\title{
Pathogenesis of cerebral malformations in perinatal spina bifida
} aperta

\author{
Olga A de Wit*1, WF den Dunnen ${ }^{2}$, KM Sollie ${ }^{3}$, OF Brouwer ${ }^{1}$ and DA Sival ${ }^{1,4}$
}

\begin{abstract}
Address: ${ }^{1}$ Department of Pediatric Neurology, University of Groningen, University Medical Centre Groningen, the Netherlands, ${ }^{2}$ Department of Pathology and Laboratory Medicine, University of Groningen, University Medical Centre Groningen, the Netherlands, ${ }^{3}$ Department of Obstetrics, University of Groningen, University Medical Centre Groningen, the Netherlands and ${ }^{4}$ Department of Pediatrics, University of Groningen, University Medical Centre Groningen, the Netherlands

Email: Olga A de Wit* - o.a.de.wit@neuro.umcg.nl

* Corresponding author
\end{abstract}

from $\left.5\right|^{\text {st }}$ Annual Meeting of the Society for Research into Hydrocephalus and Spina Bifida Heidelberg, Germany. 27-30 June 2007

Published: 20 December 2007

Cerebrospinal Fluid Research 2007, 4(Suppl I):S9 doi:I0.II86/1743-8454-4-SI-S9

This abstract is available from: http://www.cerebrospinalfluidresearch.com/content/4/SI/S9

(C) 2007 de Wit et al; licensee BioMed Central Ltd.

\section{Background}

Spina bifida aperta (SBA) is associated with cerebral morbidity, such as hydrocephalus, Chiari II malformation and cortical dysplasia. Insight in the pathogenesis of these malformations is incomplete. In fetal SBA, such information may help to improve pre- and early postnatal treatment strategies. In perinatal SBA, we investigated the time of initiation of concurrent cerebral malformations.

\section{Materials and methods}

In 7 SBA fetuses and 1 neonate [16-40 (median 28) weeks gestational age (g.a.)], we cross-sectionally investigated the histology of the aqueduct [ $\mathrm{n}=5]$, cerebral convexity and parenchyma $[\mathrm{n}=8$ ] by haematoxylin-eosin and nestin staining. The meningomyelocele was located at cervical $[\mathrm{n}=1]$, thoracic $[\mathrm{n}=3]$ and lumbar $[\mathrm{n}=4]$ spinal level. Cerebral histology was intra-individually associated with fetal ultrasound parameters (ventricular size, head circumference and Chiari II malformation). The mean and median duration between fetal ultrasound and histological assessment were both 4 days.

\section{Results}

In SBA fetuses of all gestational ages, histological malformations at the aqueduct (hemosiderophages/gliosis [5/5] and forking/slit like deformities [5/5]) were present. In the two youngest fetuses (16 and 21 weeks g.a.), we observed peri-aqueductal ependymal denudation, pro- genitor cell loss and heterotopia. From the 2nd half of pregnancy onwards, Chiari II malformation concurred with ventriculomegaly $[4 / 6]$ and successively, with macrocephaly from 37 weeks g.a. onwards [3/3]. In absence of arachnoidal fibrosis, delivery-related haemorrhages were present in all fetuses (at the fossa posterior and/or cerebrum in $6 / 7$ and 5/7 fetuses, respectively). In the only patient that succumbed during the first week after birth (39 weeks g.a.), raised intracranial pressure concurred with arachnoidal fibrosis at the convexity.

\section{Conclusion}

In fetal SBA, the earliest peri-aqueductal alterations precede the development of hydrocephalus. During the 2nd half of pregnancy, ventriculomegaly appeared unrelated to CSF malabsorption. After birth, however, CSF malabsorption may increasingly contribute to the development of high-pressure hydrocephalus. These data may implicate that peri-aqueductal ependymal denudation and progenitor cell loss occur by a mechanism independent of highpressure hydrocephalus or ventricular distention. 